Running head: INTRUSIVE FLASHBACKS IN PTSD

Solving the mystery of intrusive flashbacks in Posttraumatic Stress Disorder: Comment on Brewin (2014)

Lia Kvavilashvili

University of Hertfordshire

Address for correspondence:

Lia Kvavilashvili

Department of Psychology

University of Hertfordshire

College Lane

Hatfield, Herts, AL10 9AB

United Kingdom

Tel. +44 (0) 1707285121

Fax +44 (0) 1707285073

Email:L.Kvavilashvili@herts.ac.uk 


\begin{abstract}
In the light of current controversy about the nature of intrusions in posttraumatic stress disorder (PTSD), the review by Brewin (2013) is timely and important. It will undoubtedly stimulate further research and guide researchers' quests for understanding the nature of flashbacks in PTSD. In this commentary, I briefly summarize and discuss key points made by Brewin and elaborate on some of the reasons behind the controversy. For example, the terms "involuntary autobiographical memories,” “intrusive memories,” and “flashbacks” are often used interchangeably. I propose a taxonomy revealing the key differences across these forms of memory. If flashbacks are characteristic of PTSD patients only, then it is essential that more research targeting this population is conducted with a variety of methods. Finally, some new avenues for research to study intrusive memories and flashbacks in PTSD, using a diary method and modified trauma film paradigm, are described.
\end{abstract}

Keywords: Involuntary autobiographical memories, intrusive memories, flashbacks, hotspots, Posttraumatic Stress Disorder. 


\section{Solving the mystery of intrusive flashbacks in Posttraumatic Stress Disorder: Comment on Brewin (2013)}

Research on posttraumatic stress disorder (PTSD) has yielded important insights over the past 10 years, especially with respect to intrusive memories and flashbacks, which constitute a key symptom of this debilitating disorder. Given the complexity of the phenomenon, a variety of approaches and methods have been used ranging from interview/questionnaire studies of intrusive memories in clinical and non-clinical samples, to analysis of trauma narratives in PTSD patients and experimental analogue studies in non-clinical samples (trauma-film paradigm). This research has been instrumental in testing and developing influential theories, enhancing our understanding of mechanisms involved in the formation and maintenance of recurrent intrusions in PTSD, and contributing to various therapeutic approaches in clinical practice.

In parallel, independent of this research, remarkable progress has also been achieved in a new area of cognitive psychology on involuntary (non-intrusive) autobiographical memories in (mostly) non-clinical population, with numerous publications and several books on the topic since the publication of a pioneering study by Berntsen (1996). Recently, researchers of involuntary autobiographical memory and mainstream cognitive psychology have started to question some of the findings and theoretical assumptions of clinical research on intrusive memories in PTSD.

In the light of current controversy about the nature of intrusive memories in PTSD, the review article by Brewin (2013) is both important and timely. The major contribution of this review to the literature on intrusive memories and more generally to cognitive psychology, as I see it, is (a) making a very strong and convincing case for the existence of perceptually-based memory representation system that can be accessed both non-consciously (implicitly) and consciously (explicitly), and (b) situating research on intrusive phenomena and their theoretical explanations within the context of normal cognitive processes. It is essential, however, as 
pointed out by Brewin (2013), that we “consider how ordinary memory mechanisms may operate under usual and unusual circumstances” (p. XX). In other words, we need to explore the variables that modulate the operation of these normal processes in response to extreme levels of stress experienced by patients during traumatic events to explain the highly intrusive and repetitive nature of flashbacks and other clinical phenomena such as nightmares, hypervigilance, and partial and/or temporary amnesia for details of the traumatic event, as described in clinical cases. We also need to explain why repeated exposure to these highly distressing memories results in disappearance rather than increase of intrusions and other symptoms of PTSD.

My aim is to briefly summarize the key points of Brewin (2013), elaborate on reasons behind the controversy, discuss some of the challenges faced by research on intrusive memories in general and PTSD in particular, and outline possible avenues for future research in this area.

\section{Summary of Key Points}

\section{Long- term Perceptual Memory System}

Brewin (2013) brings together several different streams of research within cognitive and clinical psychology to support the existence of a long-term memory system that automatically encodes sensory perceptual information, and may sometimes operate separately from the more language-based episodic and autobiographical memory systems postulated by current theories of memory. Although the existence of implicit memory and priming and its underlying Perceptual Representation Memory System is now textbook material in cognitive psychology, the basic assumption, until recently, has been that this system manifests itself nonconsciously (implicitly) in behavioral responses (e.g., reaction times or word stem completions) and that its effects are relatively short lived, not lasting longer than few minutes, hours, or days, depending on the tasks and materials used. However, evidence reviewed by Brewin provides support for the idea that not only does the encoding of low-level perceptual minutiae occur automatically but that this information can subsequently be retrieved in the form of conscious 
intrusive images and memories in the presence of some potent cues, even after long periods of delay.

Evidence emerging from SenseCam research concerning long-term retention of everyday minutiae--including one’s own thoughts (which are notoriously difficult to remember, e.g., Brewer, 1988)--is promising and can potentially change current theories of episodic and autobiographical memories (e.g., see Conway, 2009). Further evidence, not reviewed in the target article, comes from initial research on involuntary semantic memories or mind-pops in both clinical and non-clinical samples, which shows that even briefly encountered novel information (e.g., a street name seen in passing) may later pop into one's mind sometimes even without person’s conscious knowledge that this information had been encountered (Kvavilashvili \& Mandler, 2004; Elua, Laws \& Kvavilashvili, 2012). This type of memory representations were referred to as "free radicals" by Tulving (1983), who proposed that they had not been integrated into either episodic or semantic memory systems and, hence, represented more of an anomaly than a standard mode of operation. In contrast, Brewin proposed (2013) the existence of long-term perceptually based memory system that is crucial in the production of various consciously experienced involuntary phenomena, including the intrusive memories and flashbacks in PTSD.

\section{Dissociation between Long-term Perceptual and Language-based Memory Systems}

Dissociations between implicit and explicit memory are well documented in cognitive memory research. Brewin (2013) reviews additional evidence from cognitive psychology that shows dissociations between the two systems (e.g., on verbal overshadowing or using SenseCam). In relation to PTSD intrusions, Brewin summarizes findings from both experimental and correlational studies, which have used the trauma-film paradigm asking participants to record subsequent intrusions and testing participants' episodic memory for scenes in the trauma film. Meta-analysis of these studies reveal that the number of recorded film intrusions do not correlate with performance on episodic memory tasks, which measure the retention of film 
content. Furthermore, although manipulations of concurrent activities during the film affected the number of recorded intrusions, the group differences were not usually mirrored by similar differences in episodic memory tasks as shown in Tables 2, 3 and 4 (for a particularly interesting dissociation, see Krans, Näring, Holmes \& Becker, 2009).

\section{Nature of Voluntary (Narrative) Memory of Traumatic Events in PTSD}

A review of the literature, presented by Brewin (2013) on impaired voluntary memory for trauma (one of the key symptoms of PTSD according to the DSM criteria), shows that all those studies in which trauma narratives were independently judged by raters provide support for clinical observations of increased fragmentation or disorganization of trauma narratives. In contrast, those studies that asked participants themselves to rate these aspects of their trauma memory (using one or two items in a questionnaire) did not find any such effects. It is therefore concluded that the results obtained will depend on the method used and that the judges' ratings

are more objective and preferable to participants' own ratings. It is, however, obvious that it will be very difficult to achieve consensus on this contentious issue. Indeed, it is difficult to study empirically the type of disorganization/fragmentation and partial or even complete amnesia for aspects of trauma event reported in clinical case studies of PTSD, especially if researchers use non-clinical or non-PTSD populations, who do not experience such symptoms.

\section{Effects of Visuospatial and Verbal Concurrent Tasks on Subsequent Intrusions}

The Dual Representation Theory assumes two separate and relatively independent routes to the formation and recalling of trauma memories: the long-term perceptually based visuospatial representation system and the language based conceptual memory system (Brewin, Dalgleish, \& Joseph, 1996). It is assumed that concurrent visuospatial and verbal tasks at the time of encoding tap into these two systems separately and produce modality-specific interference with contrasting effects on subsequent intrusions and flashbacks. In particular, if a person engages in a secondary task taxing his or her visuospatial system, this performance will interfere with the automatic formation of perceptual images of trauma and result in reduction of 
subsequent trauma-related intrusions. In contrast, engagement in a concurrent verbal activity would interfere with the processes occurring in the language-based memory system. Although logically this should lead to some impairment of person's voluntary narrative of the trauma event (perhaps making it less coherent and/or incomplete), the main and somewhat counterintuitive prediction of the theory is that concurrent verbal tasks enhance processing in visuospatial perceptual system, which will result in increased number of subsequent intrusions compared to no task control.

Brewin (2013) provides a comprehensive review of empirical studies that directly test the key predictions of the Dual Representation Theory using traumatic film clips and manipulating the nature of concurrent tasks in non-clinical samples. He provides strong support for the idea that visuospatial tasks presented during or after the trauma film, such as complex tapping task or the game Tetris, reliably reduce the number of recorded film intrusions in comparison to control conditions (without or with other concurrent tasks). Of particular interest are recent studies that managed to obtain these results by engaging participants in visuospatial tasks 30 minutes or several hours after watching the film (Deeprose, Zhang, DeJong, Dalgleish, \& Holmes, 2012; Holmes, James, Kilford, \& Deeprose, 2010).

However, one problem is that the majority of relevant studies have used relatively complex visuospatial tasks, which should also engage, to a certain degree, the central executive component of Working Memory system. Hence, it is not entirely clear whether the reduction of intrusions is due to selective engagement of the visuospatial sketchpad, the central executive, or both. For example, growing research examining the role of eye movements, while a person holds an image of negative and/or intrusive memory in mind, shows that engaging visuospatial system in this way has beneficial effects in the direction predicted by the Dual Representation Theory (Gunter \& Bodner, 2008; Lilley, Andrade, Turpin, Sabin-Farell, \& Holmes, 2009). However, this research has also shown that eye movements tax the central executive by reducing performance on a concurrent reaction time task (van den Hout, Engelhard, Rijkeboer, 
Koekebakker, Hornsvel, Leer et al., 2011). Could it be, therefore, that asking participants to generate random numbers, a task that taps primarily into central executive resources, will produce the same amount of reduction in intrusions as complex tapping task or the Tetris game? Recent findings by Pearson and Sawyer (2011) seem to provide initial support for this conjecture, even though they used emotional pictures instead of a trauma film (which could have affected the results, as pointed out by Brewin, 2013). However, Isaacs (2004) has reported similar results using the so- called Numerical Distraction Therapy with 26 PTSD patients who, as part of their therapy session, had to hold in mind their intrusive images while simultaneously completing a demanding counting task (counting backwards in 2s from 10 to 0 and then up to 10 again). It is clear that more systematic research is needed to address this important question.

In contrast to research on concurrent visual tasks, evidence concerning concurrent verbal tasks within the trauma film paradigm is mixed and appears to depend on the nature of concurrent tasks used. Two separate analyses were reported by Brewin (2013). The analyses of seven studies that used counting backwards in 3s or 7s showed that the concurrent verbal task resulted in a statistically significant increase in the number of film intrusions in two studies, a non-significant trend in the predicted direction in three studies, and a non-significant trend in the opposite direction (verbal task reducing intrusions in comparison to no task control) in two other studies. Although the overall effect, using meta-analytic procedures on these seven studies, came out as significant, it is obvious that the findings are not as straightforward as they have been with concurrent visuospatial tasks. Moreover, a separate but similar analysis of those five studies that used different verbal tasks (e.g., simple counting or remembering a 9-digit number) resulted in non-significant effects.

It is interesting that simple counting or holding a 9-digit number engages primarily the phonological loop, whereas counting backwards by 3s and 7s requires additional central executive resources, as they impair performance on simple reaction time tasks (van den Hout, Engelhard, Smeets, Hornsveld, Hoogeveen, de Heer, et al. 2010). Therefore, it is unclear to what 
extent the effects predicted by the Dual Representation Theory concerning concurrent verbal tasks also relate to engaging the central executive in addition to modality specific verbal processes within the phonological loop. It is also interesting that more cognitively based theories would predict that engaging verbal processes and counting backwards in 3s and 7s should decrease rather than increase subsequent intrusions and distress associated with them, as was indeed reported Isaacs (2004) (see also Pearson \& Sawyer, 2011; van den Hout et al., 2010). It is therefore important that future research systematically compares the predictions of the Dual Representation Theory and general theories of cognitive load and working memory, using a trauma-film paradigm with both analogue and clinical samples (see below). One potential issue that arises in relation to this paradigm, as with research on involuntary phenomena in general, is that there is a large variability in the number of recorded film intrusions, which can range from 0 to over 30 per participant (e.g., see Holmes, Brewin \& Hennessy, 2004). The data are therefore non-normally distributed and may be affected by one or two outliers in some but not other conditions. Consequently, it is important to examine such data for outliers and if necessary, exclude them from analyses or use statistical procedures that are sensitive to the (almost always) non-normal distribution of intrusion errors.

\section{Reasons Behind the Controversy}

Several important reasons underlying the nearly continuous debates between clinical and cognitive psychologists are discussed by Brewin (2013). Here, I would like to elaborate on several additional issues, which in my view, have also contributed to the controversy and confusion about intrusive memories in PTSD.

\section{Problems with Terminology and Definition of Intrusive Memories}

A key problem is confusion about the terminology used, and what is actually studied, involuntary autobiographical memories, intrusive memories, or flashbacks. These terms are often used interchangeably even though they refer to different phenomena. According to the Diagnostic and Statistical Manual of Mental Disorders (DSM-IV), the PTSD diagnosis requires 
that "the traumatic event is persistently re-experienced" (Criterion B), which may consist of "recurrent and distressing recollections of the event” (B1), and/or “acting or feeling as if the traumatic event was recurring (includes the sense or reliving the experience, illusions, hallucinations and dissociative flashback episodes)” (B3). From this description, it is obvious that the types of intrusive recollections described as B1 and B3 are quite different. Although B1 refers to what is typically called 'intrusive memories,' which are also experienced by general population and people with clinical disorders (e.g., patients with depression), B3 refers to a very special type of intrusive phenomena, associated with an intense sense of current threat (as if the trauma is recurring) and involves, in extreme cases, complete loss of sense of current reality and control of one's situation. ${ }^{1}$ These flashbacks appear to be a key characteristic feature of PTSD only (Brewin et al., 1996; Reynolds \& Brewin, 1998).

Despite these important differences between intrusive memories and flashbacks, many published studies use these terms interchangeably. The situation is exacerbated by the fact that in some studies researchers have started using the term "involuntary autobiographical memory" when referring to intrusive memories and vice versa (e.g., Brewin, 1998; Bywaters, Andrade, Turpin, 2004; Deeprose et al., 2012), presumably because intrusiveness is equated with the spontaneity of recall.

By clear definition of each of these concepts, confusion and controversy can be greatly reduced. Indeed, involuntary autobiographical memories are memories of past events that pop into mind unexpectedly without any attempt to recall anything, often in response to external or internal triggers. They may affect one’s current mood (Berntsen \& Hall, 2004; Kvavilashvili \& Schlagman, 2011). They may refer to positive, negative, or completely neutral events such as remembering one’s first romantic kiss, a car accident, or bumping into a neighbor at a supermarket. In contrast, intrusive memories are spontaneous involuntary memories of a (mostly) negative event, which repeatedly intrude upon consciousness, often against one’s will, are hard to control, and may disrupt one's ongoing activities. Although these intrusive memories 
may sometimes be of positive events (e.g., Brewin, Christodoulides, \& Hutchinson, 1996; Bywaters et al., 2004), they are predominantly negative and disturbing.

Although ordinary involuntary autobiographical memories and intrusive memories are similar in terms of their spontaneous nature, they are different in a number of ways. Indeed, everyday involuntary autobiographical memories are random, one-off memories, which pop into mind only once or twice and may never be recalled again, whereas the key feature of intrusive memories is their repetitive nature, so that a particular memory or image keeps coming back and disturbing the individual. Moreover, everyday involuntary autobiographical memories, even if negative, do not normally disrupt ongoing activities. Often, it is hard to even notice them, as they form part of a normal stream of consciousness and do not stay in mind for longer than few seconds. Of importance, they do not involve active avoidance attempts, which is one of the key features of intrusive memories.

It is even more important to distinguish clearly intrusive memories and trauma flashbacks in PTSD (see Brewin et al., 1996; Reynolds \& Brewin, 1999). Although intrusive memories can in principle be about a positive or a negative event, this is not the case for flashbacks, which by definition refer to traumatic events involving high levels of intense fear, helplessness, and horror (e.g., when being threatened by a knife and raped, witnessing death or causing someone's death, etc.). The most important difference between the two lies in the nature of reliving of the event in memory. Even though intrusive memories often induce bodily reactions and feelings associated with the original event, the person is still aware of this being a recollective experience or "mental time travel” that is characteristic of autobiographical memories in general. In contrast, the re-experiencing in flashbacks is different from reliving in intrusive memories as it involves an overwhelming sense of "nowness” accompanied by a strong startle response and high degrees physiological arousal. As a result, Brewin et al. (1996) characterized flashbacks more as spontaneous re-enactments than as just reliving of trauma (p. 671). One of the difficulties of distinguishing intrusive memories from flashbacks is that 
flashbacks can vary "from relatively mild (there is a transient sense of event re-occurring in the present) to severe (the person loses all connection with his or her current self and surroundings while re-experiencing the memory)” (p. 211, Brewin, Gregory, Lipton, \& Burgess, 2010).

The three types of memories described above can be presented on a continuum with involuntary autobiographical memories and flashbacks occupying its opposite poles and intrusive memories in the middle, sharing some features with each (see Figure 1). The important question that arises is whether the difference between intrusive memories and flashbacks (especially the relatively mild ones with only transient sense of nowness) is quantitative or qualitative. According to clinical research and the Dual Representation Theory, the difference is qualitative, whereas cognitive psychologists argue that it is quantitative (Berntsen, 2001; Rubin, Boales \& Berntsen, 2008). Answering this important question is difficult but not impossible. It may require more targeted behavioral studies on PTSD participants (see below) as well as input from fMRI and lesion studies (e.g., Koenigs \& Grafman, 2009; Whalley, Kroes, Huntley, Rugg, Davis \& Brewin, 2013).

\section{Problems with Studying PTSD Flashbacks in Analogue Studies}

Clinical observations and case studies suggest that PTSD patients have a small number of recurring images and/or memories about their trauma, only a few of which have the qualities of flashback type re-enactment/re-experiencing that is specifically characteristic of PTSD (e.g., Holmes, Grey \& Young, 2005). Therefore, the existing controversy may also be related to the implicit assumption that the Dual Representation Theory was proposed to explain all intrusions in PTSD rather than a relatively small subset of them (i.e., flashbacks).

However, Brewin et al. (1996) clearly distinguished two types of intrusive memories in PTSD. (1) Intrusive memories, which are essentially part of normal autobiographical memory system and can be recalled both deliberately in the form of trauma narrative and as recurrent intrusive memories (see also Reynolds \& Brewin, 1999). Such intrusive memories are often associated with secondary emotions of sadness, anger, or guilt and can occur in the general 
population and other clinical samples (e.g., people with depression). (2) In contrast, flashbacks can only be recalled involuntarily, in response to internal or external triggers, and consist predominantly of visual perceptual images.

If intrusive trauma memories and flashbacks are distinguished in this way, there appears to be no real contradiction between general cognitive theories of autobiographical memory and the Dual Representation Theory, as they are simply trying to explain different types of phenomena. This formulation, however, contains paradoxes that will need to be addressed and clarified. First, if flashbacks are special form of intrusive memories, based entirely on the longterm perceptual representation system, then analogue research with non-clinical samples cannot be generalized to flashbacks occurring in PTSD. Instead, as pointed out by Brewin (2013), research on flashbacks should be conducted on PTSD patients who are actually suffering from these flashbacks. The second paradox revolves around the assumption that patients' most disturbing and intrusive flashbacks cannot be accessed voluntarily. This is strange, given that in therapy, patients are asked to deliberately recall the most distressing parts of their trauma memory, which patients appear to be able to do (e.g., Isaacs, 2004; see also Brewin, Huntley, \& Whalley, 2012; Holmes et al., 2005). If patients cannot voluntarily access their flashback, this would mean that they have no conscious awareness of it after they cease experiencing it. In other words, inability to voluntarily recall a particular flashback would imply the existence of amnesia for the content of this flashback until a person encounters a potent cue that spontaneously triggers the memory. Although patients have been reported to have amnesia for some details of the traumatic event, normally in the course of therapy they are able to provide some description of their most disturbing and recurring flashback type intrusions. Moreover, several studies have asked PTSD participants to provide a written narrative of their trauma event and then to retrospectively indicate which parts of the trauma narrative elicited flashback experience(s). Participants were able to do so (see Brewin et al., 2012; Hellawell \& Brewin, 2002, 2004). Having indicated such places in their narrative, patients should be able to deliberately bring into 
their mind the same flashbacks, if requested. In light of this evidence, it would be important to have some discussion in the literature about this aspect of trauma flashback-including whether it manifests in therapy and in everyday life as specified by the Dual Representation Theory.

\section{Avenues for Future Research}

These are clearly interesting times for research on intrusive memories and flashbacks in PTSD. The growing number of empirical studies and theorizing in this field, as well as the controversy between clinical and more cognitively oriented researchers, indicates that we may be on a verge of important breakthroughs and developments in near future. The key to these developments, in my view, is adopting a broader approach, as advocated by Brewin (2013), and bringing together various strands of research that have been developing relatively independently. These include research on involuntary autobiographical memories, on the one hand, and research on intrusive memories and flashbacks, on the other. In addition, there is growing research situated within the Working Memory model examining the effects of concurrent verbal and visuospatial tasks on the vividness and emotionality of negative memory images (mostly in non-clinical samples). Below, I conclude by outlining some additional avenues for future research.

\section{Studying Flashbacks in PTSD Patients}

Irrespective of the methodology used and of researchers' orientation (clinical or cognitive), future studies need to concentrate to a greater degree on clinical samples with PTSD to study the actual phenomenon in question: the flashbacks. There is already evidence that it is possible to achieve do so, for example, by asking PTSD patients to provide a detailed written trauma narrative and retrospectively indicate which parts of the narrative (called hotspots) brought about uncontrollable visual flashbacks when writing the narrative (e.g., Holmes et al., 2005). Two studies have used interesting variations of this method. Brewin et al. (2012) exposed PTSD patients to words and phrases from their own or other patients' trauma narratives and examined which cues elicited flashback intrusions. Michael, Ehlers, Halligan, and Clark (2005) 
used the so-called "intrusion provocation task" by exposing assault victims to pictures depicting various assaults (bought from press agencies), then assessing whether these pictures elicited intrusive memories from their own assault. Both methods elicited reports of flashbacks in patients, which indicates that intrusive flashbacks, despite their unpredictable nature, can be studied in PTSD patients in much more focused and systematic fashion than previously thought.

Moreover, the success of these methods indicates that the predictions of the Dual Representation Theory can be directly tested on PTSD patients, in addition to analogue samples, by using a modified version of a trauma-film paradigm. Indeed, trauma film clips can be tailored in such a way that they depict several different incidents that are thematically similar to patient sample’s own trauma (e.g., road traffic accidents versus assaults, depending on a composition of the sample tested). Immediately after watching the film, participants report which parts of the film elicited flashbacks or intrusions of their own trauma, if any, and briefly describe the content of these flashbacks. In the subsequent week, PTSD participants keep a diary of their own (and/or film) intrusions elicited by the film and the effects of concurrent tasks during and/or after the film can be assessed. Of particular interest would be to examine whether the completion of concurrent visual tasks during the film reduce the likelihood of experiencing one's own trauma flashbacks and the concurrent verbal tasks increase the chances of such flashbacks ( $c f$. Hellawell \& Brewin, 2002).

\section{Diary Studies of Intrusive Memories and Flashbacks in PTSD}

Research on intrusive memories and flashbacks can benefit from using a diary method, which is one of the key methodological tools in research on involuntary autobiographical memories and has consistently produced valid and replicable findings. It should enable the gathering of comparable data on intrusive memories and flashbacks. One important question that has not been addressed, and can be studied with a diary method, is to examine the nature of ordinary (non-trauma) involuntary autobiographical memories, repetitive intrusive (but non flashback) memories of trauma, and trauma flashbacks in PTSD patients by asking them to keep 
a diary of these memories (using a within- or between-subjects design). It is, for example, unclear what role ordinary involuntary autobiographical memories play in the maintenance of PTSD symptoms. Is it possible that they are likely to be linked (remotely or indirectly) to the traumatic event (e.g., Berntsen, 2001)—and could this further enhance negative mood or elicit intrusions about the trauma itself? Another interesting issue that this research could clarify is to examine whether the same memory image can sometimes occur as an intrusive memory (without the sense of nowness) and as a full-blown flashback re-enactment, in which the patient feels as if the traumatic event is happening again.

\section{Comparing Predictions of Dual Representation Theory and Working Memory Model}

It is particularly urgent to systematically investigate and compare the contrasting predictions of Dual Representation Theory and Working Memory Model in order to answer the following question: Do we have modality-specific interference effects on the number of reported intrusions with concurrent visuospatial and verbal tasks, or are the obtained effects at least partly due to more general interference by taking up some central executive resources, as initially demonstrated by Pearson and Sawyer (2011)? Or are both modality- specific and general interference at play (Kemps \& Tiggerman, 2007)? Another important question is to find whether the effects of concurrent tasks are mediated by working memory span of participants. For example, is it possible that the interference effects with concurrent tasks are more likely to occur in participants with low rather than high working memory span? (e.g., Gunter \& Bodner, 2008). All these interesting questions await further investigation, and the review of Brewin (2013) will undoubtedly inform and stimulate this future research conducted by both clinical and cognitive psychologists. Together, researchers can solve the mystery of intrusive flashbacks in PTSD. 


\section{References}

Berntsen, D. (1996). Involuntary autobiographical memories. Applied Cognitive Psychology, 10, 455-460. doi: 10.1002/(SICI)1099-0720(199610)10:5<435::AIDACP408>3.0.CO;2-L.

Berntsen, D. (2001). Involuntary memories of emotional events: Do memories of traumas and extremely happy events differ? Applied Cognitive Psychology, 15, S135158. doi: $10.1002 /$ acp. 838

Berntsen, D. \& Hall, N. M. (2004). The episodic nature of involuntary autobiographical memories. Memory \& Cognition, 32, 789-803. doi: 10.3758/BF03195869.

Brewer, W. F. (1988). Memory for randomly sampled autobiographical events. In U. Neisser, \& W. Winograd (Eds.). Remembering reconsidered: ecological and traditional approaches to the study of memory (pp. 21-30). Cambridge: Cambridge University Press.

Brewin, C. R. (1998). Intrusive autobiographical memories in depression and posttraumatic stress disorder. Applied Cognitive Psychology, 12, 359-370. doi: 10.1002/(SICI)1099-0720(199808)12:4<359::AID-ACP573>3.0.CO;2-5.

Brewin, C. R., Christodoulides, J., \& Hutchinson, G. (1996). Intrusive thoughts and intrusive memories in a non-clinical sample. Cognition and Emotion, 10, 107-112. doi: 10.1080/026999396380411.

Brewin, C. R., Dalgleish, T., \& Joseph, S. (1996). A dual representation theory of post-traumatic stress disorder. Psychological Review, 103, 670-686. doi: 10.1037/0033-295X.103.4.670.

Brewin, C.R., Gregory, J. D., Lipton, M., \& Burgess, N. (2010). Intrusive images in psychological disorders: Characteristics, neural mechanisms, and treatment implications. Psychological Review, 117, 210-232. doi: 10.1037/a0018113. 
Brewin, C. R., Huntley, Z., \& Whalley, M. G. (2012). Source memory errors associated with reports of posttraumatic flashbacks: A proof of concept study. Cognition, 124, 234-238. doi: 10.1016/j.cognition.2012.05.002.

Bywaters, M., Andrade, J., \& Turpin, G. (2004). Intrusive and non-intrusive memories in a non-clinical sample: The effects of mood and affect on imagery vividness. Memory, 12, 467-478. doi: 10.1080/09658210444000089.

Conway, M. A. (2009). Episodic memories. Neuropsychologia, 47, 2305-2313. doi: 10.1016/j.neuropsychologia.2009.02.00.

Deeprose, C., Zhang, S., DeJong, H., Dalgleish, T., \& Holmes, E. A. (2012). Imagery In the aftermath of viewing a traumatic film: Using cognitive tasks to modulate the Development of involuntary memory. Journal of Behavior Therapy and Experimental Psychiatry, 43, 758-764. doi: 10.1016/j.jbtep.2011.10.008.

Elua, I., Laws, K. \& Kvavilashvili, L. (2012). From mind-pops to hallucinations? A study of involuntary semantic memories in schizophrenia. Psychiatry Research, 196, 165170. doi: 10.1016/j.psychres.2011.11.026.

Gunter, R. W., \& Bodner, G. E. (2008). How eye movements affect unpleasant memories: Support for a working-memory account. Behaviour Research and Therapy, 46, 913-931. doi: 10.1016/j.brat.2008.04.006.

Hellawell, S. J., \& Brewin, C. R. (2002). A comparison of flashbacks and ordinary autobiographical memories of trauma: cognitive resources and behavioural observations. Behaviour Research and Therapy, 40, 1143-1156. doi: 10.1016/S0005-7967(01)00080-8.

Hellawell, S. J., \& Brewin, C. R. (2004). A comparison of flashbacks and ordinary autobiographical memories of trauma: content and language. Behaviour Research and Therapy, 42, 1-12. doi: 10.1016/S0005-7967(03)00088-3. 
Holmes, E. A., Brewin, C. R., \& Hennessy, R. G. (2004). Trauma films, information processing, and intrusive memory development. Journal of Experimental Psychology: General, 133, 3-22. doi: 10.1037/0096-3445.133.1.3.

Holmes, E. A., Brewin, C. R., \& Grey, (2005). Intrusive images and “'hotspots”' of trauma memories in Posttraumatic Stress Disorder: an exploratory investigation of emotions and cognitive themes. Journal of Behaviour Therapy and Experimental Psychiatry, 36, 3-17. doi: 10.1016/j.jbtep.2004.11.002.

Holmes, E. A., James, E. L., Kilford, E. J., \& Deeprose, C. (2010). Key steps in developing a cognitive vaccine against traumatic flashbacks: Visuospatial Tetris versus verbal pub quiz. Plos One, 5. doi: 10.1371/journal.pone.0013706.

van den Hout, M. A., Engelhard, I. M., Smeets, M. A. M., Hornsveld, H., Hoogeveen, E., de Heer, E., Toffolo, M. B. J., \& Rijkeboer, M. (2010). Counting during recall: Taxing of working memory and reduced vividness and emotionality of negative memories. Applied Cognitive Psychology, 24, 303-311. doi: 10.1002/acp.1677. van den Hout, M. A., Engelhard, I. M., Rijkeboer, M. M., Koekebakker, J., Hornsveld, H., Leer, A., Toffolo, M. B. J., \& Aske, N. (2011). EMDR: Eye movements superior to beeps in taxing working memory and reducing vividness of recollections. Behaviour Research and Therapy, 49, 92-98. doi: 10.1016/j.brat.2010.11.003.

Isaacs, J. S. (2004). Numerical distraction therapy: Initial assessment of a treatment for posttraumatic stress disorder. Traumatology, 10, 39-54. doi: $10.1177 / 153476560401000104$.

Kemps, E., \& Tiggemann, M. (2007). Reducing the vividness and emotional impact of distressing autobiographical memories: The importance of modality-specific interference. Memory, 15, 412-422. doi: 10.1080/09658210701262017. 
Koenigs, M., \& Grafman, J. (2009). Post-traumatic stress disorder: The role of medial prefrontal cortex and amygdala. Neuroscientist, 15, 540-548. doi: $10.1177 / 1073858409333072$.

Krans, J., Näring, G., Holmes, E. A., \& Becker, E. S. (2009). Tell me more: Can a memory test reduce analogue traumatic intrusions? Behaviour Research and Therapy, 47, 426-430. doi: 10.1016/j.brat.2009.01.009.

Kvavilashvili, L. \& Mandler, G. (2004). Out of one’s mind: A study of involuntary semantic memories. Cognitive Psychology, 48, 47-94. doi: 10.1016/S00100285(03)00115-4.

Kvavilashvili, L. \& Schlagman, S. (2011). Involuntary autobiographical memories in dysphoric mood. Memory, 19, 331-345. doi:

10.1080/09658211.2011.568495.

Lilley, S. A., Andrade, J., Turpin, G., Sabin-Farrell, R., \& Holmes, E. A. (2009). Visuospatial working memory interference with recollections of trauma. British Journal of Clinical Psychology, 48, 309-321. doi: 10.1348/014466508X398943.

Michael, T., Ehlers, A., Halligan, S. L., \& Clark, D. M. (2005). Unwanted memories of assault: what intrusion characteristics are associated with PTSD? Behaviour Research and Therapy, 43, 613-628. doi: 10.1016/j.brat.2004.04.006.

Pearson, D. G., \& Sawyer, T. (2011). Effects of dual task interference on memory intrusions for affective images. International Journal of Cognitive Therapy, 4, 122-133. doi: 10.1521/ijct.2011.4.2.122.

Reynolds, M., \& Brewin, C. R. (1998). Intrusive cognitions, coping strategies and emotional responses in depression, post-traumatic stress disorder and a non-clinical population. Behaviour Research and Therapy, 36, 135-147. doi: 10.1016/S00057967(98)00013-8. 
Reynolds, M., \& Brewin, C. R. (1999). Intrusive memories in depression and posttraumatic stress disorder. Behaviour Research and Therapy, 37, 201-215. doi: 10.1016/S0005-7967(98)00132-6.

Rubin, D. C., Boals, A., \& Berntsen, D. (2008). Memory in posttraumatic stress disorder: Properties of voluntary and involuntary, traumatic and nontraumatic autobiographical memories in people with and without posttraumatic stress disorder symptoms. Journal of Experimental Psychology: General, 137, 591-614. doi: 10.1037/a0013165.

Schlagman, S. \& Kvavilashvili, L. (2008). Involuntary autobiographical memories in and outside the laboratory: How different are they from voluntary autobiographical memories? Memory and Cognition, 36, 920-932. doi: 10.3758/MC.36.5.920.

Tulving, E. (1983). Elements of episodic memory. Oxford: Oxford University Press.

Whalley, M. G., Kroes, M. C. W., Huntley, Z., Rugg, M. D., Davis, S. W., \& Brewin, C. R. (2013). An fMRI investigation of posttraumatic flashbacks. Brain \& Cognition, 81, 151159. doi: 10.1016/j.bandc.2012.10.002. 


\section{Footnote}

${ }^{1}$ It is important that in DSM-5, a similar distinction is drawn between B1 and B3 with B1 defined as "Recurrent, involuntary, and intrusive memories" and B3 as "Dissociative reactions (e.g., flashbacks) which may occur on a continuum from brief episodes to complete loss of consciousness”. 


\section{Acknowledgements}

The commentary has benefitted from discussions with Jamie Hacker Hughes on intrusive memories and from useful comments by Diana Kornbrot on earlier draft of the manuscript. 


\begin{tabular}{|c|c|c|c|}
\hline & $\begin{array}{l}\text { Involuntary } \\
\text { Autobiographical } \\
\text { Memories }\end{array}$ & $\begin{array}{l}\text { Intrusive } \\
\text { Memories }\end{array}$ & 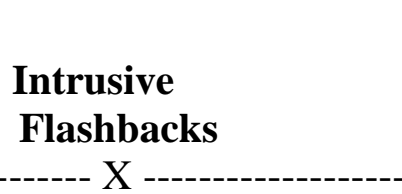 \\
\hline Retrieval & Spontaneous & Spontaneous & Spontaneous \\
\hline Repetition & Low to none & High to very high & High to very high \\
\hline Valence & Positive, Negative, Neutral & Negative, Positive & Negative \\
\hline Vividness & Low to very high & Very high & Very high \\
\hline Avoidance & None & High & High \\
\hline Disruption & None or minimal & Significant & Significant to extreme \\
\hline Distress & None to moderate & Moderate to high & High to very high \\
\hline $\begin{array}{l}\text { Re-experiencing } \\
\text { event now }\end{array}$ & No & No & Yes \\
\hline Population & Normal and clinical & Normal and clinical & PTSD only \\
\hline
\end{tabular}

Figure 1. Schematic representation of different types of involuntary memories presented on a continuum and their characteristics 